\title{
Tumor-Related Epilepsy and Post-Surgical Outcomes: Tertiary Hospital Experience in Saudi Arabia
}

\author{
Alawi A Al-Attas ${ }^{1 *}$, Hindi N Al-Hindi ${ }^{2}$, Tariq AbaAlkhail ${ }^{1}$, Amen Bawazir ${ }^{3}$, Hesham Aldhalaan $^{1}$, Ibrahim Althubaiti ${ }^{1}$ \\ and Salah Baz ${ }^{1}$ \\ ${ }^{1}$ Department of Neuroscience, King Faisal Specialist Hospital and Research Center, Saudi Arabia \\ ${ }^{2}$ Department of Pathology, King Faisal Specialist Hospital and Research Center, Saudi Arabia \\ ${ }^{3}$ College of public health and health Informatics, King Saud Bin Abdulaziz University for Health Sciences, Saudi Arabia
}

Submission: November 05, 2021; Published: November 19, 2021

*Corresponding author: Alawi A Al-Attas, Department of Neuroscience, King Faisal Specialist Hospital and Research Center, Postal Code: 11525 Riyadh P. 0. Box: 59046, Prince Sultan Military Medical City

Postal Code: 11159 Riyadh. Po Box 7897 Riyadh, Saudi Arabia

Abstract

Objectives: Recent studies reported that tumor histopathology plays a significant role in predicting freedom from seizures after epilepsy surgery; however, no consensus among researchers regarding this issue exists. This study retrospectively examined different types of tumorrelated epilepsy and post-epilepsy surgical outcomes and the relationship between various tumor Histopathology and these outcomes.

Methods: In this hospital-based retrospective study, patients with brain tumors and drug-resistant epilepsy, which is defined as the failure of two tolerated and appropriately chosen antiepileptic drugs (whether as monotherapies or in combination) to achieve and alleviate sustained seizure freedom, were recruited. These patients underwent a thorough pre-surgical evaluation in an Epilepsy Monitoring Unit (EMU) before deciding to undergo surgical intervention according to an epilepsy case management conference.

Results: One-hundred patients (including 45 children) with brain tumors were included in this study (male: female = 3:2). Most of the patients (93) had G/GNT. No significant differences in outcome were observed among sex, age, or histopathological categories. However, during the first year after epilepsy surgery, most of the low-grade G/GNT cases showed favorable outcomes based on ILAE classes 1 and 2 (61.3\% and $9.7 \%$, respectively), whereas high-grade gliomas and meningothelial tumors showed outcomes of ILAE class 1 (40\% and $100 \%$, respectively).

Conclusion: Post-epilepsy surgical outcomes of different brain tumors have been achieved with favorable outcomes in children and adults with low-grade gliomas and meningiomas. Thus, pre-surgical evaluation in EMU is highly recommended to enhance better post-epilepsy surgical outcomes.

Keywords: Epilepsy; Brain tumor; Histopathology; Surgery; Saudi Arabia; ILAE

\section{Introduction}

Approximately $30 \%-50 \%$ of patients with brain tumors have epilepsy as an initial presentation [1]. However, 6\%-45\% of patients with brain tumors develop seizures later in life [2,3]. Although the exact mechanism of seizure development in patients with brain tumors is not clearly understood, recent studies have identified that some changes in the peritumoral regions affect the release of neurotransmitters that lead to seizure development [4]. Many studies have reported a relationship between tumor type and seizure frequency. For example, low-grade gliomas and glioneuronal tumors (G/GNT) are associated with a high rate of seizure incidence (85\%-92\%) [5-7]. In contrast, glioblastomas, which are high-grade tumors, are associated with a low rate of seizure incidence $(20 \%-50 \%)[8,9]$. The incidence rates of seizures in meningiomas, especially in atypical and malignant subtypes, remain understudied [10]. Despite the abundance of knowledge regarding surgical management of resistant forms of epilepsy, including those associated with brain tumors, especially of the low-grade type, prospective studies regarding the medical treatment of epilepsy in this type of patient are scarce [11]. Reportedly, resection of the epileptogenic zone due to the development of brain tumors lead to freedom from or significant control of seizures in $70-90 \%$ of patients $[12,13]$. Furthermore, 
tumor type, seizure severity, early surgical intervention, frequency during the pre-operative stage, histopathology of the tumors, and the extension of surgical resection to include peritumoral tissues are reportedly the factors that increase the likelihood of freedom from seizures post-operatively [14]. Two of the best predictors of freedom from post-operative seizures include a duration of less than one year since the onset of epilepsy and gross total surgical resection $[15,16]$. Tumor histopathology plays a significant role in predicting freedom from seizures after epilepsy surgery; however, there is no consensus among researchers regarding this issue $[17,18]$. This study retrospectively investigated different types of tumor-related epilepsy and their outcomes in the first year after epilepsy surgery.

\section{Materials and Methods}

\section{Study design}

A hospital-based retrospective study was conducted using secondary data from the epilepsy registry at King Faisal Specialist Hospital and Research Center (KFSH\&RC) between 1998 and 2017.

\section{Study population}

The included patients underwent surgery for drug-resistant epilepsy. The patients were admitted to the Epilepsy Monitoring Unit (EMU) for long-term monitoring; they underwent presurgical evaluations such as surface electroencephalography (EEG), 3-tesla magnetic resonance imaging (MRI) of the brain, and fluorodeoxy-glucose positron emission tomography (PET) brain scans. Additionally, a qualified neuropsychologist was present during the evaluation of the enrolled patients with epilepsy. In some patients, intracranial subdural recordings, intracarotid amobarbital procedure (Wada test) and electrocorticography (ECoG), and motor, sensory, and language mapping were performed.

Detailed information about the patients was collected. This information included demographic characteristics (age, gender, handedness, age at onset of the disease) and history and clinical data (type of seizure and frequency, seizure observed at EMU, MRI findings, PET scan, ictal EEG (IEEG) location and type, subdural EEG recording, and inter-ictal EEG [IIEEG] location and type). Moreover, final diagnosis, surgical procedures, and pathology were recorded as the primary outcome. All tumor cases enrolled in this study were reviewed and graded independently by a neuropathologist according to the World Health Organization (WHO) classification [19]. Epilepsy data were discussed in an epilepsy surgery conference with epileptologists, epilepsy surgeons, neuroradiologists, and neuropsychologists to determine the status and surgical candidacy of the patient.

According to the International League Against Epilepsy (ILAE) commission report (1997-2001), six outcomes of interest were proposed [20]. However, the categories 4, 5, and 6 are difficult to measure and implement in daily practice, particularly when parameters related to the quality of life are included. Hence, we suggested simplifying the ILAE classification of epilepsy surgical outcomes with only four categories rather than six to facilitate its application for a new modification that shares the ILAE classification for classes 1,2 , and 3 , while classes 4,5 , and 6 would be merged into only one class, called class 4 . Thus, the definitions of the classes were divided into four groups:

1. class 1 , patients who were completely seizure-free with no auras;

2. class 2, patients with auras but no seizures;

3. class 3 , patients experiencing one to three seizure days per year \pm auras; and

4. class 4 , ranging from four or more seizure days per year to those experiencing $\geq 50 \%$ reduction of baseline seizure days \pm auras to $\geq 100 \%$ increase in baseline seizure days; \pm auras. Furthermore, outcomes of classes 1 and 2 were considered favorable, while those of classes 3 and 4 were considered unfavorable. These four outcomes were investigated during the first post-surgical year.

\section{Data analysis}

Statistical analysis through cross-tabulation of the tumor groups, pathologies, and progressive outcomes was performed using SAS software (ver. 9.4). Due to the small sample size, some subgroups, pathologies, and outcomes were collapsed. Proportional statistics, and chi-square and Fisher's exact test were used to explain the findings within a $95 \%$ confidence interval (CI). A P-value of $<0.05$ was considered statistically significant.

\section{Results}

Among the 100 patients with brain tumors related epilepsy included in this study, 59 (59\%) were males, and 41 (41\%) were females. The incidence of brain tumor-related epilepsy (BTRE) in our center was lower among children than adults (45\% versus 55\%). However, the differences among sex, age, and the histopathology categories were not statistically significant $(\mathrm{P}=$ 0.111 and 0.878 , respectively) as depicted in Table 1.

According to histopathological findings, the cases of brain tumors were grouped into three main categories (Table 2). Most of the tumors $(93 \%)$ were low-grade gliomas or glioneuronal tumors (G/GNT) followed by high-grade gliomas (5\%); only two meningioma cases (2\%) were detected. Among the three main categories of brain tumors, low-grade G/GNT consisted of 11 entities. Under this category, 40 tumors were gangliogliomas, and 29 tumors were Dysembryoplastic neuroepithelial tumors (DNET). Among the five high-grade gliomas, three were astrocytic tumors. Interestingly, only two meningiomas were found. Table 2 details the pathological categories. 


\section{Open Access Journal of Neurology \& Neurosurgery}

Table 1: Histopathologies of the brain tumors and patient characteristics $(N=100)$.

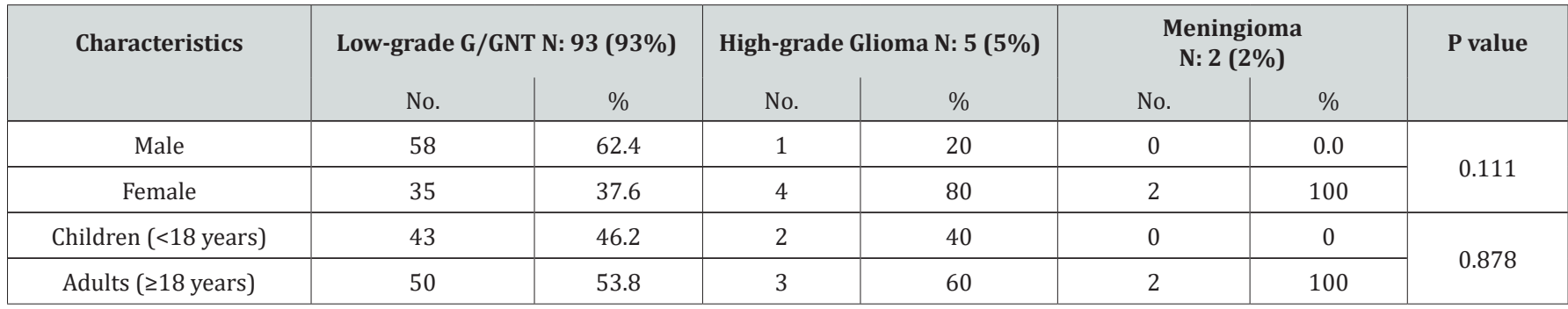

Table 2: The three main categories and the subgroups of brain tumor.

\begin{tabular}{|c|c|c|c|}
\hline Types of brain tumor & Subgroups & $\mathbf{n}$ & $\%$ \\
\hline \multirow[t]{11}{*}{ Low-grade Gliomas (93 cases) } & Ganglioglioma & 40 & 40 \\
\hline & Dysembryoplastic neuroepithelial tumor & 29 & 29 \\
\hline & Oligodendroglioma & 7 & 7 \\
\hline & Gangliocytoma & 5 & 5 \\
\hline & Oligoastrocytoma & 3 & 3 \\
\hline & Composite Ganglioglioma/Dysembryoplastic neuroepithelial tumor & 3 & 3 \\
\hline & Diffuse Astrocytoma & 2 & 2 \\
\hline & Ependymoma & 1 & 1 \\
\hline & Neurocytoma & 1 & 1 \\
\hline & Papillary glioneuronal tumor & 1 & 1 \\
\hline & Composite Pleomorphic Xanthoastrocytoma (PXA) and Ganglioglioma & 1 & 1 \\
\hline \multirow{2}{*}{ High-grade Gliomas ( 5 cases) } & Anaplastic Astrocytoma/Glioblastoma & 4 & 4 \\
\hline & Anaplastic Oligodendroglioma & 1 & 1 \\
\hline Meningiothelial tumors ( 2 cases) & Meningioma & 2 & 2 \\
\hline
\end{tabular}

Table 3: Seizure outcome one year after surgery according to histopathology.

\begin{tabular}{|c|c|c|c|c|c|c|c|c|}
\hline \multirow[t]{2}{*}{ Group } & \multicolumn{2}{|c|}{ Class 1} & \multicolumn{2}{|c|}{ Class 2} & \multicolumn{2}{|c|}{ Class 3} & \multicolumn{2}{|c|}{ Class 4} \\
\hline & No. & $\%$ & No. & $\%$ & No. & $\%$ & No. & $\%$ \\
\hline Low-grade G/GNT & 57 & 61.3 & 9 & 9.7 & 9 & 9.7 & 18 & 19.4 \\
\hline High-grade Gliomas & 2 & 40 & 1 & 20 & 1 & 20 & 1 & 20 \\
\hline Meningiomas & 2 & 100 & 0 & 0 & 0 & 0 & 0 & 0 \\
\hline
\end{tabular}

completely seizure free, no auras (2) only auras, no seizures (3) 1-3 seizure days per year \pm auras (4) $\geq 4$ seizure days per year to $\leq 50 \%$ reduction of baseline seizure days to $\geq 100 \%$ increase of baseline seizure days; \pm auras.

Table 4: Favorable and unfavorable outcomes one year after surgery.

\begin{tabular}{|c|c|c|c|c|c|}
\hline \multirow{2}{*}{$\begin{array}{c}\text { Characteristics } \\
\text { Histopathological type }\end{array}$} & \multicolumn{2}{|c|}{ Favorable outcome $n=71(71 \%)$} & \multicolumn{3}{|c|}{ Unfavorable outcome $n=29(29 \%)$} \\
\hline & No. & $\%$ & No. & $\%$ & $P$ value \\
\hline Low-grade G/GNT & 66 & 71 & 27 & 29 & 0.864 \\
\hline High-grade Gliomas & 3 & 60 & 2 & 40 & \\
\hline Meningioma & 2 & 100 & 0 & 0 & \\
\hline \multicolumn{6}{|l|}{ Age } \\
\hline Adults & 36 & 52.2 & 17 & 58.6 & 0.559 \\
\hline Children & 33 & 47.8 & 12 & 41.4 & \\
\hline \multicolumn{6}{|l|}{ Gender } \\
\hline Male & 41 & 57.8 & 18 & 62 & 0.159 \\
\hline Female & 30 & 42.2 & 11 & 38 & \\
\hline
\end{tabular}




\section{Open Access Journal of Neurology \& Neurosurgery}

In this study, the primary postoperative outcome was evaluated using the modified ILAE classification described in the methodology section. During the first post-surgical year, most patients with low-grade G/GNT experienced favorable outcomes (classes 1 and 2, 61.3\% and 9.7\%, respectively) as shown in Table 3. In contrast, approximately $40 \%$ of the patients with high-grade gliomas showed favorable outcomes (ILAE class 1$)$. Both meningioma cases $(100 \%)$ showed outcomes of ILAE class 1 . Among the 100 patients with brain tumors, 71 (71\%) patients, including 66 patients with low-grade G/GNT, three patients with high-grade gliomas, and two patients with meningiomas, experienced favorable outcomes during the oneyear post-surgical period (Table 4). Moreover, the incidence of favorable outcome was higher in adult patients than in children $(52.2 \%$ versus $47.8 \%)$ and in male versus female patients (41\% versus 30\%). However, these differences in outcome dependent on histopathological type, age, and sex were not statistically significant $(\mathrm{P}=0.864,0.559$, and 0.159 , respectively).

\section{Discussion}

From the epilepsy registry at KFSH\&RC, 100 patients who underwent surgery for tumor-related epilepsy were included in this study. Varying associations between different brain tumor types and epilepsy have been reported. Most studies show gangliogliomas to be the most common tumor type associated with epilepsy, followed by DNET, oligodendrogliomas, and astrocytomas [21-24]. These findings support our results, that is, most of our patients had low-grade G/GNT (93.0\%), most of which were gangliogliomas (40\%) followed by DNET (29\%). Furthermore, Babini et al. reported that gangliogliomas $(66.7 \%$ versus $40 \%$ ) [25] were the most frequent tumors among their patients; however, their sample size was smaller (30 cases) than in our study. Contrary to our findings, Kahlenberg et al. reported that mixed oligo-astrocytomas were the most prevalent tumors followed by astrocytomas grade II and oligodendrogliomas of grade II [25]. The incidence of high-grade gliomas was lower (5\%) in our study than in a study by Michelucci et al. in Italy (77.0\%), a finding attributed to a significantly high prevalence of high-grade gliomas in that area [15].

Seizures have a great impact on patient and caregiver quality of life. Seizures affect all aspects of a patient's life, such as employment, social life, driving, and entertainment. Epilepsy surgery as described in many recent studies, is considered to relieve tumor-related epilepsy and achieve favorable outcomes $[15,26,27]$. However, in some cases, epilepsy persist even after resecting the primary focus [25]. All of our patients underwent well-planned epilepsy surgery, which is defined as the resection of the tumor and peritumoral tissues [23]. Consequently, our patients showed varying degrees of improvement during the first postsurgical year depending on the type of brain tumor (low-grade G/ GNT versus high-grade gliomas); however, the differences were not statistically significant $(\mathrm{P}=0.864)$. For example, patients with low-grade tumors, which were the most common type of tumor in our study (93 cases), showed a favorable outcome during the first post-epilepsy surgery year with approximately $71 \%$ freedom from seizure (classes 1 and 2). Michelucci et al. reported findings that were similar to our study, that is, better outcomes was observed in patients with low-grade gliomas (76\%) [15]. In contrast, Kahlenberg et al. showed that about half of their patients (30 out of 54; 55.6\%) with brain tumor-related epilepsy showed good post-surgical outcomes (seizure-free periods $>12$ months) [25]. These proportions were lower than those observed in our study (55.6\% versus $71 \%)$.

In our opinion, the ILAE classification of epilepsy surgical outcomes should be simplified using only four categories rather than six to facilitate ILAE application. Hence, whenever patients have four or more seizures (outcomes 4,5 , and 6), they should be classified into one category (category 4). The currently used ILAE Commission on Neurosurgery in 2001[28] still has some elements that make the use of categories 4, 5, and 6 difficult to measure and implement in daily practice, particularly when including parameters related to quality of life. This new modified classification can help the researchers in their ongoing studies.

Furthermore, few patients in our study had high-grade gliomas (five) or meningiomas (two). These patients showed a favorable outcome during the first post-surgical year $(60 \%$ and $100 \%$, respectively). Michelucci et al. reported similar results in which $58 \%$ of their patients with high-grade glioma became seizure free after tumor removal [15]

Pediatric and adult groups showed no significant differences regarding seizure outcomes during the first post-surgical year (P $=0.559$ ); thus, we cannot claim that surgery is more beneficial in pediatric patients. These findings were similar to those of other studies [23].

Our study has three main limitations:

1. the sample size was small and included only five patients with high-grade gliomas and two patients with meningothelial tumors (two cases); this made comparison with low-grade G/GNT insufficient although our sample size (100 cases) is comparable to that of other studies;

2. this series of tumor-related epilepsy does not represent the population with epilepsy in Saudi Arabia because not all patients with tumor-related epilepsy are eligible to be admitted to our institution, and thus, they are treated; and

3. the possibility of bias occurring during data collection in a retrospective study. Despite the above-mentioned limitations, we hope that our study provides valuable information on one of the most debatable topics in epilepsy surgery in the country and throughout the Middle East. 


\section{Open Access Journal of Neurology \& Neurosurgery}

\section{Conclusion}

In this study, the most common tumor-related epilepsy was low-grade G/GNT. Outcomes of post epilepsy surgery of different brain tumors have been achieved with a favorable outcome in both children and adults. Thus, thorough pre-surgical evaluation of patients with brain tumor-related epilepsy in EMU is highly recommended to enhance better post-epilepsy surgical outcomes. Further prospective, multicenter studies are needed with a larger number of patients to allow the findings to be more generalizable in Saudi Arabia.

\section{Acknowledgement}

The authors gratefully acknowledge American manuscript editors for English language editing. Special gratitude to Amal Abujaber, epilepsy coordinator, for her great contribution to the study. We would also like to thank Edward Devol and Samia $\mathrm{Al} \mathrm{Hashim} \mathrm{for} \mathrm{facilitating} \mathrm{the} \mathrm{collection} \mathrm{of} \mathrm{required} \mathrm{data} \mathrm{for} \mathrm{this}$ study.

\section{Ethical approval}

The study was approved by IRB from the Office of Research Affairs in King Faisal Specialist Hospital and Research Centre (RAC \# 2151084). As this is a retrospective study, secondary data has been collected from Epilepsy registry at King Faisal Specialist Hospital and Research Centre without identification of patient's data, hence no informed consent has been required.

\section{References}

1. Van Breemen MS, Wilms EB, Vecht CJ (2007) Epilepsy in patients with brain tumours: epidemiology, mechanisms, and management. Lancet Neurol 6(5): 421-430.

2. Cowie CJ, Cunningham MO (2014) Peritumoral epilepsy: relating form and function for surgical success. Epilepsy \& Behav 38: 53-61.

3. Lee JW, Wen PY, Hurwitz S, Black P, Kesari S, et al. (2010) Morphological characteristics of brain tumors causing seizures. Arch Neurol 67(3): 336-342.

4. You G, Sha Z, Jiang T (2012) The pathogenesis of tumor-related epilepsy and its implications for clinical treatment. Seizure 21(3): 153-159.

5. Aronica E, Leenstra S, van Veelen CW, van Rijen PC, Hulsebos TJ, et al. (2001) Glioneuronal tumors and medically intractable epilepsy: a clinical study with long-term follow-up of seizure outcome after surgery. Epilepsy Res 43(3): 179-191.

6. Chang EF, Potts MB, Keles GE, Lamborn KR, Chang SM, et al. (2008) Seizure characteristics and control following resection in 332 patients with low-grade gliomas. J Neurosurg 108(2): 227-235.

7. Hamasaki T, Yamada K, Kuratsu J (2013) Seizures as a presenting symptom in neurosurgical patients: a retrospective single-institution analysis. Clin Neurol Neurosurg 115(11): 2336-2340.

8. Prakash O, Lukiw WJ, Peruzzi F, Reiss K, Musto AE (2012) Gliomas and seizures. Med Hypotheses 79(5): 622-626.

9. Rosati A, Marconi S, Pollo B, Tomassini A, Lovato L, et al. (2009] Epilepsy in glioblastoma multiforme: correlation with glutamine synthetase levels. J Neurooncol 93(3): 319-324.
10. Wang YC, Chuang CC, Tu PH, Wei KC, Wu CT, et al. (2017) Seizures in Surgically Resected Atypical and Malignant Meningiomas: Long-Term Outcome Analysis. Epilepsy res 140: 82-89.

11. James E, Varelas PN (2014) Brain Tumor and Seizures: Incidence, Pathophysiology, Diagnosis and Treatment InTech Journals 411-424.

12.Zentner J, Hufnagel A, Wolf HK, Ostertun B, Behrens E, et al. (1997) Surgical treatment of neoplasms associated with medically intractable epilepsy. Neurosurgery 41(2): 378-386.

13. Melo JGSP, Centeno RS, Malheiros SMF, Ferraz FAP, Stávale JN, et al. (2007) Clinical Features and Surgical Outcome of Patients with Indolent Brain Tumors and Epilepsy. J Epilepsy Clin Neurophysiol 13: 65-69.

14. Giulioni M, Marucci G, Martinoni M, Marliani AF, Toni F, et al. (2014) Epilepsy associated tumors: Review article. World J Clin Cases 2(11): 623-641.

15. Englot DJ, Berger MS, Barbaro NM, Chang EF (2012) Factors associated with seizure freedom in the surgical resection of glioneuronal tumors. Epilepsia 53(1): 51-57.

16. García-Fernández M, Fournier-Del Castillo C, Ugalde-Canitrot A, PérezJiménez Á, Juan Álvarez-Linera, et al. (2011) Epilepsy surgery in children with developmental tumours. Seizure 20(8): 616-627.

17. Kahlenberg CA, Fadul CE, Roberts DW, Thadani VM, Bujarski KA, et al. (2012) Seizure prognosis of patients with low-grade tumors. Seizure 21(7): 540-545.

18. Melo JG, Centeno RS, Malheiros SM, Ferraz FA, Stávale JN, et al. (2007) Clinical features and surgical outcome of patients with indolent brain tumors and epilepsy. J Epilepsy Clin Neurophysiol 13: 65-69.

19. Louis DN, Ohgaki H, Wiestler OD, Cavenee WK, Burger PC, et al. (2007) The 2007 WHO classification of tumours of the central nervous system. Acta Neuropathol 114(2): 97-109.

20. Wieser HG, Blume WT, Fish D, Goldensohn E, Hufnagel A, et al. (2001) ILAE Commission Report. Proposal for a new classification of outcome with respect to epileptic seizures following epilepsy surgery. Epilepsia 42(2): 282-286.

21. Hess KR, Broglio KR, Bondy ML (2004) Adult glioma incidence trends in the United States, 1977-2000. Cancer 101(10): 2293-2299.

22. Kemerdere R, Yuksel O, Kacira T, Yeni N, Ozkara C, et al. (2014) Lowgrade temporal gliomas: surgical strategy and long-term seizure outcome. Clin Neurol Neurosurg 126:196-200.

23. Zaatreh MM, Firlik KS, Spencer DD, Spencer SS (2003) Temporal lobe tumoral epilepsy Characteristics and predictors of surgical outcome. Neurology 61(5): 636-641.

24. Phi JH, Kim SK, Cho BK, Lee SY, Park SY, et al. (2009) Long-term surgical outcomes of temporal lobe epilepsy associated with low-grade brain tumors. Cancer 115(24): 5771-5779.

25. Babini M, Giulioni M, Galassi E, Marucci G, Martinoni M, et al. (2013) Seizure outcome of surgical treatment of focal epilepsy associated with low-grade tumors in children. J Neurosurg Pediatr 11(2): 214-223.

26. Meguins LC, Adry RARdC, Silva Júnior SCd, Pereira CU, Oliveira JGd, et al. (2015) Gross-total resection of temporal low grade gliomas is a critically important factor in achieving seizure-freedom. Arquivos de neuro-psiquiatria 73(11): 924-928.

27. Donadío M, D Giano C, Moussalli M, Barrios L, Ugarnes G, et al. (2011) Epilepsy surgery in Argentina: Long-term results in a comprehensive epilepsy centre. Seizure 20(6): 442-445.

28. Englot DJ, Chang EF, Vecht CJ (2016) Epilepsy and brain tumors. Handb Clin Neurol 134: 267. 
